\section{Curious Crystals: Chylous Arthritis in Rheumatoid Arthritis}

\section{To the Editor:}

This report describes a chylous joint effusion in a man with longstanding, untreated rheumatoid arthritis (RA). Chylous arthritis is a rare complication of RA, and frequently mimics septic joint because of the presence of a milky "purulent" joint aspirate.

A 68-year-old man was referred to the rheumatology clinic for assessment of what appeared to be inflammatory arthritis and rheumatoid nodules. He was a retired truck driver and had a history of gout, type 2 diabetes, hypertension, hyperlipidemia, coronary artery disease with past myocardial infarction, and atrial fibrillation/flutter leading to implantation of a defibrillator. He was an ex-smoker and nondrinker. His daughter had arthritis as a child, and both of his parents had a history of "rheumatism." His medications included allopurinol, aspirin/dipyridamole, fluvastatin, glyburide, lansoprazole, metformin, metoprolol, niacin, ramipril, warfarin, and magnesium.

He denied any history of arthritic pain, and expressed some confusion about why he was referred to the rheumatology clinic. He stated that he never had any pain in his hands, feet, knees, or hips. He stated that he had no morning stiffness. When asked about the large, obvious nodules on the extensor surfaces of his forearms, he stated that they had been present for about 30 years. He told us that because they did not bother him, he did not seek any medical attention.

On examination, the most marked finding was swelling in both elbows with large clumps of rheumatoid nodules in both olecranon bursae and along the extensor surface of the forearms (Figure 1). There was swelling present across the metacarpophalangeal (MCP) joints of all 10 digits. There was an early Boutonniere deformity in the left third digit. Additionally, there was a small effusion present in both the right knee and ankle. Despite pressing quite firmly on all the joints, we could not elicit any tenderness.

We aspirated the large mass of nodules present on the left elbow. We believe that the true elbow was aspirated, but given the large bursal swelling it was difficult to discern from where the fluid originated. Milky fluid was aspirated, which with time separated into globules (Figure 2). Under polarized microscopy, large negatively birefringent plates were visualized, consistent with cholesterol (Figure 3).

Because of the presence of cholesterol, we took radiographs of the elbow to rule out occult fracture. This was negative. Radiographs of the hands revealed extensive erosive changes in the MCP and carpal structures bilaterally. Radiographs of the feet showed erosions in the metatarsophalangeal and metatarsal heads. Rheumatoid factor was positive at 1:100 IU (normal < $20 \mathrm{IU}$ ) and anticyclic citrullinated peptide was $>100$ (normal < 5). Analysis of the aspirated fluid showed $18.2 \times 10^{9}$ cells/l nucleated cells

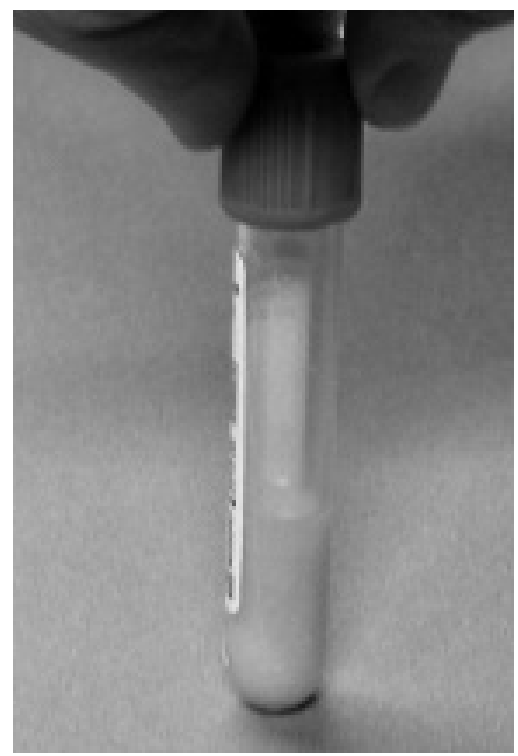

Figure 2. Aspirated fluid gradually separated into globules.

with $87 \%$ lymphocytes. The pathologist confirmed the presence of numerous cholesterol crystals

We initiated triple therapy for the treatment of longstanding RA. Because of the lack of pain, and the long duration of the nodules, the patient agreed to treatment grudgingly.

Although numerous case reports of chylous arthritis exist, the prevalence and incidence of chylous effusions remain unclear ${ }^{1-6}$. Clinically, it frequently mimics septic arthritis, with a warm, red joint and milky "purulent" joint aspirate.

Examination under polarized microscopy can distinguish an infected joint from a chylous effusion by directly visualizing cholesterol plates. These negatively birefringent crystals are typically flat rectangles with notched corners, although helical or rod-shaped crystals have also been reported $^{1,7}$. They range from 2 to $20 \mu \mathrm{m}$ in size $e^{1,7}$.

The cause of chylous effusions in RA is unknown. Chylous tenosynovitis with tendon xanthoma formation has also been reported in those with $\mathrm{RA}^{5}$.

Rarely, there are other causes of chylous arthritis. Trauma can cause such arthritis, possibly due to occult fracture with bone marrow leakage or

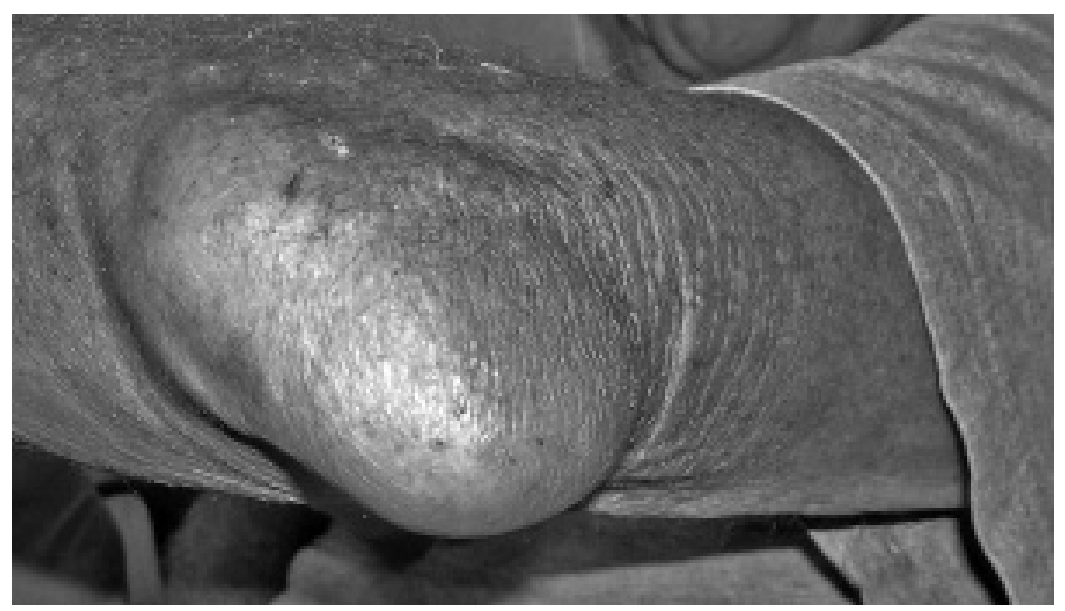

Figure 1. Swelling of the elbow with presence of many nodules. 


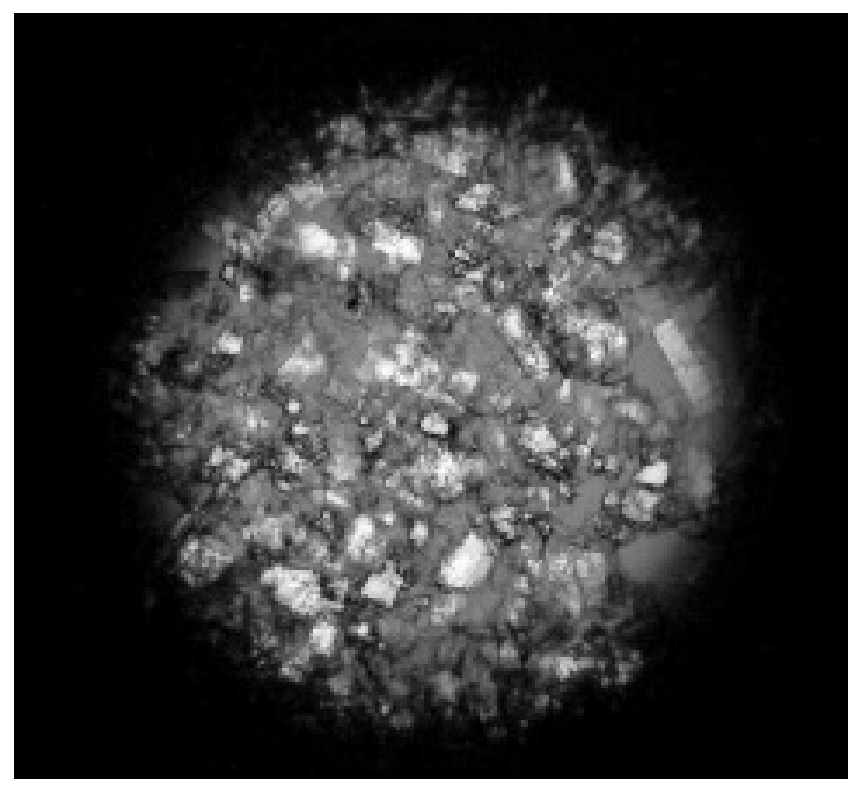

Figure 3. Under polarized microscopy, the aspirate from the swollen elbow shows multiple large, flat, negatively birefringent crystals with notched corners (original magnification $10 \times$ ).

intraarticular fat-pad injury ${ }^{6}$. Chronic filariasis is an important differential diagnosis in areas where this parasitic infection is common ${ }^{6,7}$. There are reports of chylous arthritis in patients with nephrotic syndrome and pancreatitis ${ }^{6}$.

Chylous arthritis is difficult to treat and often leads to chronic inflammation. There has been a report of successful treatment with atorvastatin ${ }^{2}$.
One case report described resolution of the effusion with appropriate treatment of the underlying $\mathrm{RA}^{3}$. Another group successfully used radiation synovectomy with yttrium- $90^{4}$.

It is important to be aware of the possibility of chylous arthritis when considering a patient with an apparent septic joint. As in this case, the milky aspirate can closely resemble bacterial infection. Demonstration of typical crystals on microscopy can help to differentiate between infectious and chylous arthritis.

SHERRY ROHEKAR, BSc, MD, FRCPC, Rheumatology Centre, St. Joseph's Hospital, Monsignor Roney Building, Level D2, 268 Grosvenor Street,

London, Ontario N6A 4V2, Canada.

E-mail: sherry.rohekar@sjhc.london.on.ca

\section{REFERENCES}

1. Jansen TL, Spoorenberg A. Medical mystery: arthritis - the answer. N Engl J Med 2006;355:421-2.

2. Jansen TL. Atorvastatin for chronic synovitis due to massive intra-articular cholesterol monohydrate deposition in long-standing rheumatoid arthritis. Rheumatology 2006;45:1577-8.

3. Ho S, Srinivasan U, Bevan M. Cholesterol crescents and plates in shoulder effusion of a rheumatoid patient. Rheumatology 2008;47:377-8.

4. Cooper AM, Pritchard MH. Chylous shoulder joint effusion treated by radiosynovectomy. Br J Rheumatol 1994;33:1201-2.

5. Balint PV, Kane D, Sturrock RD. Case number 26: massive cholesterol crystal deposition: unusual location in rheumatoid arthritis. Ann Rheum Dis 2003;62:512.

6. Soojian MG, Tejwani N. Chylous knee effusion: is it septic arthritis? A case report and review of literature. J Trauma 2004;57:1121-4.

7. Das GC, Sen SB. Chylous arthritis. BMJ 1968;2:27.

J Rheumatol 2010;37:5; doi:10.3899/jrheum.091103 